\title{
Population impact of different definitions of airway obstruction
}

\author{
B.R. Celli*, R.J. Halbert", , S. Isonaka*, B. Schau ${ }^{+}$
}

Population impact of different definitions of airway obstruction. B.R. Celli, R.J. Halbert, S. Isonaka, B. Schau. (C) ERS Journals Ltd 2003.

ABSTRACT: There is currently no consensus on the criteria for diagnosing chronic obstructive pulmonary disease. This study evaluated the impact of different definitions of airway obstruction on the estimated prevalence of obstruction in a population-based sample.

Using the Third National Health and Nutrition Examination Survey, obstructive airway disease was defined using the following criteria: 1) self-reported diagnosis of chronic bronchitis or emphysema; 2) forced expiratory volume in one second (FEV1)I forced vital capacity (FVC) $<\mathbf{0 . 7 0}$ and FEV1 $<\mathbf{8 0} \%$ predicted (Global Initiative for Chronic Obstructive Lung Disease (GOLD) Stage IIA); 3) FEV1/FVC below the lower limit of normal; 4) FEV 1/FVC $<88 \%$ pred in males and $<89 \%$ pred in females; 5) FEV1/ FVC $<0.70$ ("fixed ratio"). Spirometry in this dataset did not include reversibility testing, making it impossible to distinguish reversible from irreversible obstruction.

Rates in adults varied from 77 per 1,000 (self-report) to 168 per 1,000 (fixed ratio). For persons aged $>\mathbf{5 0}$ yrs, the fixed ratio criteria produced the highest rate estimates. For all subgroups tested, the GOLD Stage II criteria produced lower estimates than other spirometry-based definitions.

Different definitions of obstruction may produce prevalence estimates that vary by $>\mathbf{2 0 0} \%$. International opinion leaders should agree upon a clear definition of chronic obstructive pulmonary disease that can serve as a population-based measurement criterion as well as a guide to clinicians.

Eur Respir J 2003; 22: 268-273.

\begin{abstract}
*Dept of Medicine, Tufts University School of Medicine, Boston, MA, "\#Constella Health Strategies, Santa Monica, and "Dept of Community Health Sciences, UCLA School of Public Health, Los Angeles, CA, USA. ${ }^{+}$Dept of Market Access, Pricing \& Outcomes Research, Boehringer Ingelheim, GmbH, Ingelheim am Rhein, Germany.
\end{abstract}

Correspondence: R.J. Halbert, 2400 Broadway, Suite 100, Santa Monica, CA 90404, USA. Fax: 13103157432

E-mail: rhalbert@constellagroup.com

Keywords: Chronic obstructive pulmonary disease

prevalence

spirometry

USA

Received: August 142002

Accepted after revision: March 252003

This study was supported by Boehringer Ingelheim, $\mathrm{GmbH}$.
Chronic obstructive pulmonary disease (COPD) is the fourth leading cause of death in the USA [1]. COPD is the only leading cause of death that is increasing in prevalence worldwide [2], and is widely underdiagnosed in the primary care setting [3]. Since early detection and intervention is currently the best approach to reducing the burden of COPD, there has been a call for global strategies to assess the prevalence of COPD [2]. Unfortunately, there is no consensus on the criteria for diagnosis of COPD. However, all international bodies, as well as a majority of national guidelines, agree that COPD should be defined physiologically using spirometry $[4,5]$. The ratio of forced expiratory volume in one second (FEV1) to vital capacity (VC) or forced vital capacity (FVC) is an accepted indicator of the presence of airflow limitation. Using criteria based upon this measure, VIEGI et al. [6] demonstrated that the measured prevalence of disease depends upon the criterion used to define obstruction. Thus, the comparison of rates between countries, indeed even between individual published reports, is complicated by the differing criteria used to measure obstruction [7]. For population-based estimates to be comparable across different populations, there should be minimal systematic bias between criteria. In addition, successful widespread implementation of spirometry requires the obtained measurement be simple, reproducible and usable across the spectrum of populations to be evaluated.

Using several widely used definitions of airway obstruction, the impact of these definitions on overall prevalence estimates for the USA was compared using the Third National Health and Nutrition Examination Survey (NHANES III). Their impact on the relationship between obstruction and age, sex, race/ethnicity, and smoking status was then examined. Since the spirometric testing used in NHANES III did not include reversibility testing, it was not possible to distinguish reversible from irreversible obstruction.

\section{Materials and methods}

\section{Study subjects}

The NHANES III was conducted from 1988-1994 by the US National Center for Health Statistics. Using a stratified multistage clustered probability sample, the survey was designed to identify an unbiased sample that could, using appropriate weights, be extrapolated to the entire civilian noninstitutionalised population in the USA. Study participants completed extensive household and personal health questionnaires, and underwent a comprehensive physical examination, including spirometry. A total of 81 sites were included in the final sample. A complete description of the NHANES III survey methodology has been previously published [8].

Spirometry procedures were based on the 1987 recommendations of the American Thoracic Society (ATS) [9]. A customised Ohio Sensorimed 827 dry rolling seal spirometer (Ohio Medical Instrument Company, Cincinatti, OH, USA) was used. After calibration of the equipment, each subject performed five to eight forced expiratory manoeuvres, with the goal of meeting the ATS acceptability and reproducibility criteria [10]. A more detailed description of the spirometric procedures is available [11]. 
Among the total NHANES III sampled population, 13,322 patients had a reliable and reproducible spirometry test. This population represented a weighted reference population of $147,438,632$ people, which included persons aged $\geqslant 17 \mathrm{yrs}$ of all races. For the analysis, a lower age cut-off of 30 yrs was used in order to reduce the confounding effects of asthma in younger people. Subjects aged $>80 \mathrm{yrs}$, and subjects not of Caucasian, African-American or Mexican-American race/ethnicity were excluded because there were no reliable reference equations for spirometry results. Thus, the study population included non-Hispanic Whites, non-Hispanic Blacks, and Mexican-Americans aged 30-80 yrs with a satisfactory spirometry test. The total final (crude) study population was 9,838 , representing a weighted study population of $109,483,437$ subjects.

\section{Methods}

Four widely used spirometry-based definitions of airway obstruction and one self-reported definition were compared (table 1). Age, sex, and race-specific values for predicted FEV1 and predicted FEV1/FVC were calculated for each patient using reference equations described by HANKINSON et al. [10]. Similarly, age, sex, and race-specific lower limits of the normal range for FEV1/FVC were calculated for each person using reference equations from the same source. These were based on the predicted value minus 1.645-times the SE of the estimate.

Subjects who answered "yes" to the questions, "Has a doctor ever told you that you had chronic bronchitis?" or "Has a doctor ever told you that you had emphysema?" were considered to have self-reported COPD. Subjects were defined as smokers if they answered "yes" to the question, "Do you smoke cigarettes now?". Subjects who answered "no" to the question, "Do you smoke cigarettes now?" but "yes" to the question, "Have you smoked at least 100 cigarettes during your entire life?" were classified as former smokers. Persons answering "no" to both questions were labeled never-smokers. These questions were part of the NHANES III Household Adult Questionnaire.

\section{Analysis}

For all five possible definitions of airway obstruction, prevalence rates were calculated for the entire study population, and by subgroups defined by age, sex, race/ethnicity, and smoking status. Rates were weighted to the general USA population using the "total Mobile Examination Center and Home-Examined Final weight" (WTPFHX6) defined by NHANES III. Since the goal of this study was to evaluate the impact of different scenarios using a single dataset, the authors did not perform statistical testing.

\section{Results}

Table 2 describes the characteristics of the study population. For the entire sample, the mean \pm SD age was $48.3 \pm 13.6 \mathrm{yrs}$, height was $169.2 \pm 7 \mathrm{~cm}$, and weight was $77.6 \pm 18.1 \mathrm{~kg}$. FEV1 was $2.9 \pm 0.87 \mathrm{~L}$, with a FEV1/FVC of $77.0 \pm 9.0 \%$. For the $\mathrm{FEV} 1 / \mathrm{FVC} \%$ predicted, mean \pm SD was $96.6 \pm 10.2$. The values for the mean $\mathrm{FEV} 1 / \mathrm{FVC} \%$ pred minus 1.64 residual SD were $79.9 \%$ for the entire sample, $78.1 \%$ for males, and $81.7 \%$ for females.

As shown in table 3, the four spirometry-based definitions yielded estimates of total prevalent airway obstruction varying from 8.6 million (Global Initiative for Chronic Obstructive Lung Disease (GOLD) Stage IIA) to $>18$ million (fixed ratio), as compared with 8.5 million with a patient-reported history of COPD. Rate estimates varied from 79 per 1,000 population (GOLD Stage IIA) to 168 per 1,000 with the fixed ratio definition.

For persons aged $<50 \mathrm{yrs}$, the "per cent predicted" and "lower limit of normal" definitions produced the highest rate estimates. For persons aged $\geqslant 55$ yrs, the "fixed ratio" definition produced the highest rate estimates. For all age groups, the GOLD Stage IIA definition produced lower estimates than other spirometry-based definitions. Overall, the GOLD Stage IIA definition was more similar to self-reported COPD than were other spirometry-based definitions. The age relationships are presented in figure 1 .

For subgroups defined by sex, race/ethnicity and smoking status, rate estimates tended to cluster into two groups, with self-report and GOLD IIA definitions generally producing lower estimates, and the other definitions generally producing higher estimates.

\section{Discussion}

By comparing commonly used methods, it is possible to quantify the impact of different measurement approaches on population estimates of obstructive airway disease. As shown by the results from this study, these differences may be large, altering population prevalence estimates of COPD by $>200 \%$. This affects public health decisions, such as priority setting, resource allocation, and planning. In some regions, the estimated prevalence of a disease may determine the availability of additional resources or better access for patients.

These differences are also important when comparing published studies using different COPD definitions, or in comparing COPD prevalence rates between populations with

Table 1.-Definitions of airway obstruction

\begin{tabular}{|c|c|}
\hline Definition & Reference \\
\hline $\begin{array}{l}\text { Self-reported diagnosis of chronic bronchitis } \\
\text { or emphysema "self report" }\end{array}$ & See text for description of variables used \\
\hline FEV $1 /$ FVC $<0.70$ and FEV $1<80 \%$ pred $^{\#}$ & GOLD Stage IIA [12] \\
\hline FEV $1 /$ FVC $<$ lower limit of normal & See text for comparability to ATS guidelines [13] \\
\hline $\begin{array}{l}\text { FEV } 1 / F V C<88 \% \text { pred in males and }<89 \% \text { pred } \\
\text { in females "per cent predicted" }\end{array}$ & See text for comparability to ERS guidelines [14] \\
\hline FEV1/FVC $<0.70$ "fixed ratio" \# & "Clinical" definition [6], equivalent to GOLD Stage I [12] \\
\hline
\end{tabular}


Table 2.-Population characteristics

\begin{tabular}{|c|c|c|c|c|c|c|c|c|}
\hline & Crude & Weighted $^{\#}$ & FEV1 mL & FEV $1 \%$ pred & FVC mL & $\mathrm{FVC} \%$ pred & FEV1/FVC $\%$ & FEV1/FVC $\%$ pred \\
\hline Total study population & 9838 & 109483437 & $2900 \pm 866$ & $95.7 \pm 16.7$ & $3762 \pm 1038$ & $99.6 \pm 15.1$ & $77.0 \pm 9.0$ & $96.6 \pm 10.2$ \\
\hline \multicolumn{9}{|l|}{ Age yrs } \\
\hline $30-34$ & 1542 & 18978806 & $3484 \pm 777$ & $100.0 \pm 12.6$ & $4254 \pm 979$ & $102.8 \pm 12.3$ & $82.3 \pm 6.2$ & $98.2 \pm 7.2$ \\
\hline $35-39$ & 1400 & 17419936 & $3307 \pm 755$ & $98.7 \pm 12.7$ & $4083 \pm 970$ & $101.2 \pm 12.9$ & $81.4 \pm 6.0$ & $98.3 \pm 7.0$ \\
\hline $40-44$ & 1320 & 16797329 & $3228 \pm 734$ & $98.1 \pm 13.2$ & $4069 \pm 951$ & $101.2 \pm 13.1$ & $79.7 \pm 6.4$ & $97.6 \pm 7.7$ \\
\hline $45-49$ & 871 & 12041677 & $3056 \pm 744$ & $96.1 \pm 15.1$ & $3933 \pm 963$ & $99.5 \pm 13.7$ & $78.0 \pm 7.4$ & $97.0 \pm 9.1$ \\
\hline $50-54$ & 777 & 9354798 & $2856 \pm 765$ & $93.0 \pm 16.6$ & $3767 \pm 1005$ & $97.0 \pm 14.6$ & $76.2 \pm 8.0$ & $96.0 \pm 9.9$ \\
\hline $55-59$ & 734 & 8811569 & $2676 \pm 724$ & $91.9 \pm 16.9$ & $3600 \pm 965$ & $96.6 \pm 15.1$ & $74.7 \pm 8.3$ & $95.4 \pm 10.4$ \\
\hline $60-64$ & 940 & 7913347 & $2525 \pm 716$ & $92.0 \pm 19.1$ & $3452 \pm 934$ & $97.4 \pm 16.7$ & $73.4 \pm 9.3$ & $95.1 \pm 11.9$ \\
\hline $65-69$ & 827 & 7681211 & $2407 \pm 704$ & $92.6 \pm 20.1$ & $3331 \pm 937$ & $98.0 \pm 18.1$ & $72.6 \pm 9.3$ & $95.4 \pm 12.0$ \\
\hline $70-74$ & 805 & 6123341 & $2248 \pm 662$ & $92.8 \pm 20.7$ & $3186 \pm 901$ & $98.1 \pm 17.7$ & $71.2 \pm 10.5$ & $95.0 \pm 13.8$ \\
\hline $75-80$ & 622 & 4361424 & $2013 \pm 641$ & $94.0 \pm 22.8$ & $2899 \pm 881$ & $99.3 \pm 19.6$ & $70.1 \pm 11.5$ & $95.1 \pm 15.4$ \\
\hline \multicolumn{9}{|l|}{ Sex } \\
\hline Male & 4705 & 53264661 & $3367 \pm 847$ & $95.0 \pm 16.9$ & $4439 \pm 917$ & $98.6 \pm 14.2$ & $75.5 \pm 9.5$ & $96.1 \pm 11.0$ \\
\hline Female & 5133 & 56218776 & $2472 \pm 630$ & $96.3 \pm 16.6$ & $3141 \pm 699$ & $100.6 \pm 15.9$ & $78.4 \pm 8.3$ & $97.1 \pm 9.4$ \\
\hline \multicolumn{9}{|l|}{ Race/ethnicity } \\
\hline Mexican-American & 2575 & 4868314 & $3028 \pm 820$ & $98.0 \pm 14.6$ & $3823 \pm 977$ & $103.7 \pm 14.6$ & $79.2 \pm 7.6$ & $98.2 \pm 8.3$ \\
\hline Non-Hispanic Black & 2735 & 11234876 & $2745 \pm 797$ & $97.1 \pm 17.2$ & $3498 \pm 940$ & $100.2 \pm 15.7$ & $78.5 \pm 8.8$ & $96.4 \pm 9.9$ \\
\hline Non-Hispanic White & 4528 & 93380248 & $2921 \pm 916$ & $93.5 \pm 17.3$ & $3887 \pm 1098$ & $97.0 \pm 14.5$ & $74.9 \pm 9.5$ & $95.9 \pm 11.2$ \\
\hline \multicolumn{9}{|l|}{ Smoking status } \\
\hline Never & 4313 & 45490508 & $2850 \pm 839$ & $99.0 \pm 15.2$ & $3592 \pm 1016$ & $101.3 \pm 15.0$ & $79.4 \pm 7.4$ & $99.0 \pm 8.5$ \\
\hline Former & 2846 & 33672918 & $2941 \pm 880$ & $94.8 \pm 17.2$ & $3907 \pm 1034$ & $99.1 \pm 14.9$ & $75.0 \pm 9.5$ & $95.8 \pm 10.9$ \\
\hline Current & 2679 & 30320012 & $2938 \pm 889$ & $91.3 \pm 17.5$ & $3882 \pm 1037$ & $97.5 \pm 15.2$ & $75.4 \pm 10.0$ & $93.6 \pm 11.0$ \\
\hline
\end{tabular}

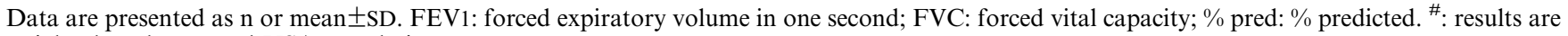
weighted to the general USA population.

different age distributions or smoking rates. Indeed, if these two factors are changing over time (as they are in the USA), there may be systematic artificial differences between measurements made at different times, even within the same country. This can yield biased trend estimates, which may mislead population projections or public health planning efforts.

Since 1991, the ATS has discouraged defining obstruction based upon a fixed ratio between the FEV1 and the FVC because this ratio is inversely related to both age and height [15]. However, the more recent 1995 guidelines of the ATS do not provide a definitive cut-off that is amenable to creating a reproducible algorithm for evaluating populations. The European Respiratory Society (ERS), on the other hand, has explicitly specified a definition of obstruction that may be applied to populations. The definition of obstruction "assessed

Table 3. - Airway obstruction by diagnostic definition

\begin{tabular}{|c|c|c|c|c|c|}
\hline & Self-report & GOLD stage IIA & Lower limit of normal & $\%$ pred & Fixed ratio \\
\hline Prevalent cases & 8.5 & 8.6 & 15.6 & 17.5 & 18.4 \\
\hline \multicolumn{6}{|l|}{ Rate per 1000 population } \\
\hline Overall & 77.3 & 78.7 & 142.1 & 160.0 & 167.8 \\
\hline \multicolumn{6}{|l|}{ Age yrs } \\
\hline $30-34$ & 49.3 & 17.3 & 83.7 & 90.4 & 44.7 \\
\hline $35-39$ & 39.5 & 18.2 & 92.5 & 100.1 & 54.6 \\
\hline $40-44$ & 65.6 & 35.7 & 115.8 & 127.1 & 94.8 \\
\hline $45-49$ & 77.1 & 50.2 & 138.8 & 152.5 & 133.5 \\
\hline $50-54$ & 86.8 & 102.5 & 156.1 & 178.8 & 181.9 \\
\hline $55-59$ & 92.3 & 137.6 & 191.8 & 212.1 & 255.6 \\
\hline $60-64$ & 109.4 & 152.4 & 197.7 & 234.4 & 311.5 \\
\hline $65-69$ & 124.0 & 179.3 & 212.5 & 256.1 & 345.4 \\
\hline $70-74$ & 137.0 & 189.0 & 228.6 & 258.3 & 406.2 \\
\hline $75-80$ & 121.9 & 194.8 & 227.2 & 261.8 & 416.9 \\
\hline \multicolumn{6}{|l|}{ Sex } \\
\hline Male & 58.2 & 84.8 & 150.0 & 160.9 & 199.0 \\
\hline Female & 95.5 & 72.9 & 134.5 & 159.2 & 138.3 \\
\hline \multicolumn{6}{|l|}{ Race/Ethnicity } \\
\hline Mexican-American & 35.7 & 27.2 & 91.5 & 87.0 & 73.2 \\
\hline Non-Hispanic Black & 58.0 & 58.5 & 119.6 & 144.8 & 116.8 \\
\hline Non-Hispanic White & 81.8 & 83.8 & 147.4 & 165.6 & 178.9 \\
\hline \multicolumn{6}{|l|}{ Smoking Status } \\
\hline Never & 45.9 & 36.0 & 72.4 & 86.8 & 90.5 \\
\hline Former & 86.4 & 89.0 & 156.6 & 178.9 & 213.9 \\
\hline Current & 114.4 & 131.2 & 230.4 & 248.8 & 232.6 \\
\hline
\end{tabular}

Prevalent cases are presented as n (millions). Results are weighted to the general USA population. GOLD: Global Initiative for Chronic Obstructive Lung Disease; $\%$ pred: $\%$ predicted. 


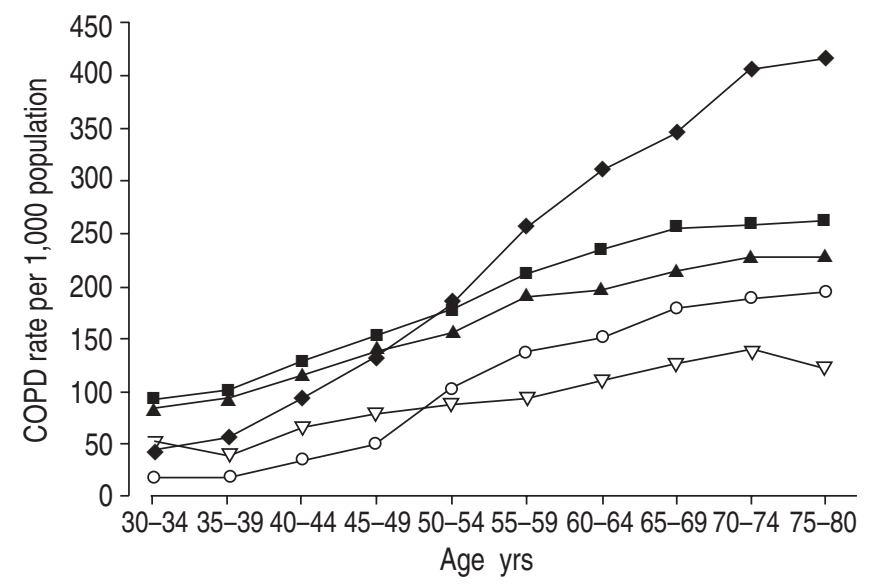

Fig. 1.-Prevalence of airway obstruction, by diagnostic definition and age group. COPD: chronic obstructive pulmonary disease. fixed ratio; $\boldsymbol{\square}$ : \% predicted; $\boldsymbol{\Delta}$ : lower limit of normal; $\bigcirc$ : Global Initiative for Chronic Obstructive Lung Disease stage IIA; $\nabla$ : self-report.

as $\mathrm{FEV} 1 / \mathrm{VC}<88 \%$ predicted in men or $<89 \%$ predicted in women" is followed by the parenthetical statement, "...i.e. $>1.64$ residual standard deviation below predicted value" [14]. Results from this study are not directly comparable to the ERS guidelines for reasons discussed in more detail below.

The recently published GOLD guidelines define COPD in subjects with an FEV1/FVC $<0.70$ [12]. This fixed ratio definition of obstruction may offer some benefits as a compromise solution among competing definitions, as it provides rate estimates that are generally comparable to those obtained using the "lower limit of normal" and "per cent predicted" methods for general population estimates as well as for subgroups of sex, race/ethnicity, or smoking status. The fixed ratio is easy to remember and does not require the use of population-specific reference tables. These qualities may lend themselves to practical use for primary care screening in all parts of the world. They also make it less daunting for the nonspecialist to interpret pulmonary function tests, which might increase the likelihood of testing in persons with respiratory symptoms. Since underdiagnosis is arguably one of the major barriers to more effective treatment of COPD [3], the widespread adoption of a definition that is "sensitive, specific, reproducible, and relatively uncomplicated to administer" [4] is desirable. However, the potential benefits of using this simplified method do not come without cost. In the results from this study, the fixed ratio produced higher rate estimates for older persons than any other definition, and these differences increased with age as the FEV1/FVC ratio declines. For the purposes of screening, a higher sensitivity may be acceptable in older, higher-risk patients. This increased sensitivity must be balanced against the increased cost of spirometry among false positives incorrectly identified as having COPD. However, the fixed ratio most closely approximates the rate produced by the lower limit of normal definition in the highest-risk subgroup, current smokers.

The GOLD Stage IIA definition (FEV1/FVC $<0.70$ and FEV $1<80 \%$ pred) is often used as a "practical" threshold for identifying COPD. When applied to the NHANES III data, this definition consistently provided the lowest rates among the spirometry-based methods. The similarity between the rates obtained using GOLD Stage IIA and self-report suggests that this may indeed be a practical definition for identifying persons with symptomatic disease.

Without a definitive "gold standard," the NHANES III dataset does not show which definition is most likely to reflect the "true" rate of COPD. Because of differences in methodology (mainly the exclusion of persons aged $<30 \mathrm{yrs}$ ), results from this study are slightly higher than prior analyses of the NHANES III dataset. Based on the GOLD Stage IIA criteria, NHANES III has yielded population estimates of $6.8 \%$ among subjects aged $\geqslant 17$ yrs [16], and $6.6 \%$ in subjects aged $\geqslant 25$ yrs (age-adjusted to the 2000 USA population) [17], compared with $7.9 \%$ in the current study. For GOLD Stage I criteria, a rate of $13.9 \%$ has been previously reported [18], compared with $16.8 \%$ with the "fixed ratio" definition used in this study. Outside the USA, published COPD prevalence estimates based on spirometry have ranged 3.7-11.0\% [7].

Using a definition that included asthma, the NHANES III prevalence of self-reported obstructive lung disease in persons aged $\geqslant 17$ yrs was $12.5 \%$ in smokers, $9.4 \%$ in former smokers, and $5.8 \%$ in never-smokers [16], compared with 11.4, 8.6, and $4.6 \%$, respectively, in the current study. Self-reported rates derived from another national-level survey in the USA, the National Health Information Survey, consistently range 5.6-6.4\% [17]. These compare well with prevalence estimates based on patient-reported disease from outside the USA, which range $3.7-10.7 \%$ [7]. The authors initially assumed that self-reported disease was unlikely to be confounded by age, sex, or smoking status. However, a recently published evaluation of respiratory symptom questionnaires in Norway suggests that self-report may indeed be differentially affected by smoking status [19].

Regardless of the "true" baseline prevalence of COPD, findings from the current study suggest that differences in age distribution, racial composition, and smoking prevalence between countries can result in systematic differences in prevalence rates when international comparisons are being made using different definitions. These results emphasise the need for an internationally agreed-upon definition of COPD, one which may serve as a population-based criterion as well as a guide to clinicians. Ideally, such a definition should be unambiguous, unbiased, and reproducible in different settings.

The reference equations derived by HANKINSON et al. [10] have been shown to perform well when compared to previously published reference populations [20]. However, more recent work suggests small but systematic differences between the NHANES III equations and other standards [21]. Of note, using the NHANES III equations resulted in fewer FEV1/FVC abnormalities indicative of obstruction than using equations by CRAPO et al. [22] or KNUDSON et al. [23]. Many of these older standards were based on smaller samples than the NHANES III, and were drawn from single regions. For example, the widely used equations published by CRAPO et al. [22] in 1981 were derived from 251 healthy nonsmokers from Salt Lake City, UT, USA. Of these, $>90 \%$ of these were Mormons. KNUDSON et al. [23] based their equations on 697 White, non-Hispanic nonsmokers living in Tucson, AZ, USA. The NHANES III equations, on the other hand, are based on 7,429 healthy nonsmokers drawn from a population-based sample of ambulatory civilian residents in the USA [10].

Results from this study are not directly comparable to the ERS guidelines, since FVC was used rather than VC measured as "slow" vital capacity (SVC), or inspiratory vital capacity (IVC), as recommended by the ERS. The 1993 ERS report on "Lung Volumes and Forced Ventilatory Flows" states that "the FVC may be considerably less than the IVC in patients with airflow limitation..." [24]. Unfortunately, neither SVC nor IVC are available in the NHANES III dataset. Despite the known differences between slow and forced measures of $\mathrm{VC}$, the relationship between FEV1/FVC and FEV1/ $\mathrm{VC}$, particularly the measurement of differences in the \% pred values for these two ratios, has not been well characterised using large population datasets. Studies using small groups of patients (i.e. $<100)$ have been published by several authors 
[25-27]. The most robust comparison is probably that of PISTELli et al. [28], which found systematic differences in reference equations for FEV1/FVC and FEV1/VC based on a population of 1,039 normal subjects. However, the effect of these differences on population estimates has not been studied and it is not clear how results from the current study would compare to results using slow VC.

The spirometry examination performed in NHANES III did not include a test of reversibility of obstruction. While the authors expect that a significant proportion of respondents had some reversible component (i.e. "mixed disease"), some subset of the study population also had pure asthma, that is, completely reversible obstruction. Without detailed diagnostic and severity information, it is not possible to determine how large a role reversible obstruction played in these findings. However the primary study goal of the authors was not the measured prevalence of COPD (i.e. the absolute number), but rather the relative difference in prevalence estimates between differing diagnostic criteria. Spirometric definitions of COPD generally require postbronchodilator measurement of lung parameters. The GOLD definition of COPD, for example, explicitly specifies, "all FEV1 values refer to a postbronchodilator FEV1" [12]. While differences in the prevalence of completely reversible obstruction may have introduced some bias between the different definitions, reversible disease is unlikely to completely explain the between-definition differences seen in findings from the current study.

International opinion leaders should agree upon a clear definition of chronic obstructive pulmonary disease that can serve as a population-based measurement criterion as well as a guide to clinicians. This definition should be simple and capable of identifying patients likely to suffer from the disease. This concept has been very useful in the clinical and epidemiological management of hypertension. Despite the complexities of actual blood pressure distributions, the use of a fixed lower limit for systolic and diastolic blood-pressures contributed to significant advances in the treatment of this disease and in the prevention of its complications, including myocardial infarction and strokes [29-31]. By reaching consensus, opinion leaders could provide comparable benefits in the area of chronic obstructive pulmonary disease.

Acknowledgements. The authors would like to thank J. Thomasson and R. Nordyke for programming support, and the editors and anonymous reviewers for important comments on the manuscript.

\section{References}

1. U.S. Department of Health and Human Services, National Institutes of Health. National Heart, Lung, and Blood Institute. Morbidity and Mortality. 2000 Chart Book on Cardiovascular, Lung, and Blood Diseases (2000). http://www.nhlbi.nih.gov/resources/docs/cht-book.htm. Date accessed: 30 April 2002.

2. Hurd SS. International efforts directed at attacking the problem of COPD. Chest 2000; 117: Suppl. 2, 336-338.

3. Voelkel NF. Raising awareness of COPD in primary care Chest 2000; 117: Suppl. 2, 372-375.

4. Calverley PMA. COPD: early detection and intervention. Chest 2000; 117: Suppl. 2, 365-371.

5. Iqbal A, Schloss S, George D, Isonaka S. Worldwide guidelines for chronic obstructive pulmonary disease: a comparison of diagnosis and treatment recommendations. Respirology 2002; 7: 233-239.

6. Viegi G, Pedreschi M, Pistelli F, Di Pede F, Baldacci S,
Carrozzi L, Giuntini C. Prevalence of airways obstruction in a general population: European Respiratory Society vs American Thoracic Society definition. Chest 2000; 117: Suppl. 2, 339-345.

7. Halbert RJ, Isonaka S, George D, Iqbal A. Interpreting COPD prevalence estimates: what is the true burden of disease? Chest 2003; 123: 1684-1692.

8. U.S. Department of Health and Human Services, National Center for Health Statistics. Plan and operation of the Third National Health and Nutrition Examination Survey, 1988-94 (1994). http://www.cdc.gov/nchs/data/series/sr_01/sr01_032.pdf. Date accessed: 20 December 2002.

9. American Thoracic Society. Standardization of spirometry an update: statement of the American Thoracic Society. Am Rev Resp Dis 1987; 136: 1285-1298.

10. Hankinson JL, Odencrantz JR, Fedan KB. Spirometric reference values from a sample of the general U.S. population. Am J Respir Crit Care Med 1999; 159: 179-187.

11. U.S. Department of Health and Human Services, National Center for Health Statistics. Third National Health and Nutrition Examination III Spirometry Procedure Manual (1988). http://www.cdc.gov/nchs/data/nhanes/nhanes3/cdrom/ NCHS/MANUALS/SPIRO.PDF. Date accessed: 20 December 2002.

12. World Health Organization, National Heart, Lung, and Blood Institute. Global Initiative for Chronic Obstructive Lung Disease. Global strategy for the diagnosis, management, and prevention of chronic obstructive pulmonary disease (2001). http://www.goldcopd.com. Date accessed: 30 April 2002.

13. American Thoracic Society. Standards for the diagnosis and care of patients with chronic obstructive pulmonary disease. Am J Resp Crit Care Med 1995; 152: Suppl. 5, 77-121.

14. Siafakas NM, Vermeire P, Pride NB, et al. on behalf of the Task Force. Optimal assessment and management of chronic obstructive pulmonary disease (COPD). Eur Respir J 1995; 8: 1398-1420.

15. American Thoracic Society. Lung function testing: selection of reference values and interpretative strategies. Am Rev Respir Dis 1991; 144: 1202-1218.

16. Mannino DM, Gagnon RC, Petty TL, Lydick E. Obstructive lung disease and low lung function in adults in the United States: data from the National Health and Nutrition Survey, 1988-1994. Arch Intern Med 2000; 160: 1683-1689.

17. Mannino DM, Homa DM, Akinbami LJ, Ford ES, Redd SC. Chronic obstructive pulmonary disease surveillance - United States, 1971-2000. MMWR Surveill Summ 2002; 51: 1-16.

18. Mannino DM. Epidemiology, prevalence, morbidity and mortality, and disease heterogeneity. Chest 2002; 121: Suppl. $5,121-126$.

19. Brogger JC, Bakke PS, Gulsvik A. Comparison of respiratory symptoms questionnaires. Int J Tuberc Lung Dis 2000; 4: 83-90.

20. Glissmeyer EW, Crapo R, Jensen R, Greenway L. Comparison of NHANES III spirometry reference equations with four previous studies (Abstract). Am J Respir Crit Care Med 1999; 159: A834.

21. Gildea TR, McCarthy K, Kavuru MS. Evaluation of spirometry reference standard equations in a clinical setting and critical appraisal of the lower limit of normal (Abstract). Am J Respir Crit Care Med 2002; 165: A200.

22. Crapo RO, Morris AH, Gardner RM. Reference spirometric values using techniques and equipment that meet ATS recommendations. Am Rev Respir Dis 1981; 123: 659-664.

23. Knudson RJ, Lebowitz MD, Holberg CJ, Burrows B. Changes in the normal maximal expiratory flow-volume curve with growth and aging. Am Rev Respir Dis 1983; 127: 725-734.

24. Quanjer PH, Tammeling GJ, Cotes JE, Pedersen OF, Peslin R, Yernault JC. Lung volumes and forced ventilatory flows. Report Working Party Standardization of Lung Function Tests, European Community for Steel and Coal. Official 
Statement of the European Respiratory Society. Eur Respir $J$ 1993; 6: Suppl. 16, 5-40.

25. Gove RI, Shepherd J, Burge PS. Variability and reversibility of the slow and forced vital capacity in chronic airflow obstruction. Br J Dis Chest 1987; 81: 182-185.

26. Chhabra SK. Forced vital capacity, slow vital capacity, or inspiratory vital capacity: which is the best measure of vital capacity? J Asthma 1998; 35: 361-365.

27. Brusasco V, Pellegrino R, Rodarte JR. Vital capacities in acute and chronic airway obstruction: dependence on flow and volume histories. Eur Respir J 1997; 10: 1316-1320.

28. Pistelli F, Bottai M, Viegi G, et al. Smooth reference equations for slow vital capacity and flow-volume curve indexes. Am J Respir Crit Care Med 2000; 161: 899-905.
29. Ibrahim M, Chobanian AV, Horan M, Roccella EJ Hypertension prevalence and the status of awareness, treatment, and control in the United States. Hypertension 1985; 7: 457-468.

30. MacMahon S, Rodgers A. The effects of blood pressure reduction in older patients: an overview of five randomized controlled trials in elderly hypertensives. Clin Exper Hypertens 1993; 15: 967-978.

31. Neal B, MacMahon S, Chapman N. Blood Pressure Lowering Treatment Trialists' Collaboration. Effects of ACE inhibitors, calcium antagonists, and other bloodpressure-lowering drugs: results of prospectively designed overviews of randomised trials. Lancet 2000; 356: 19551964. 